\title{
JNK contributes to temozolomide resistance of stem-like glioblastoma cells via regulation of MGMT expression
}

\author{
MASASHI OKADA $^{1}$, ATSUSHI SATO ${ }^{1,2}$, KEITA SHIBUYA $^{1}$, ERIKO WATANABE ${ }^{1,5,6}$, \\ SHIZUKA SEINO ${ }^{1,5,6}$, SHUHEI SUZUKI ${ }^{1,3}$, MANABU SEINO ${ }^{1,4}$, YOSHITAKA NARITA ${ }^{7}$, \\ SOICHIRO SHIBUI $^{7}$, TAKAMASA KAYAMA ${ }^{2}$ and CHIFUMI KITANAKA ${ }^{1,5,6}$ \\ Departments of ${ }^{1}$ Molecular Cancer Science, ${ }^{2}$ Neurosurgery, ${ }^{3}$ Clinical Oncology, and ${ }^{4}$ Obstetrics \\ and Gynecology, Yamagata University School of Medicine; ${ }^{5}$ Oncology Research Center, \\ Research Institute for Advanced Molecular Epidemiology, Yamagata University, Yamagata 990-9585; \\ ${ }^{6}$ Global COE Program for Medical Sciences, Japan Society for Promotion of Science, Tokyo 102-8471; \\ ${ }^{7}$ Department of Neurosurgery and Neuro-Oncology, National Cancer Center Hospital, Tokyo 104-0045, Japan
}

Received September 1, 2013; Accepted October 18, 2013

DOI: 10.3892/ijo.2013.2209

\begin{abstract}
While elimination of the cancer stem cell population is increasingly recognized as a key to successful treatment of cancer, the high resistance of cancer stem cells to conventional chemoradiotherapy remains a therapeutic challenge. $\mathrm{O}^{6}$-methylguanine DNA methyltransferase (MGMT), which is frequently expressed in cancer stem cells of glioblastoma, has been implicated in their resistance to temozolomide, the first-line chemotherapeutic agent against newly diagnosed glioblastoma. However, much remains unknown about the molecular regulation that underlies MGMT expression and temozolomide resistance of glioblastoma cancer stem cells. Here, we identified JNK as a novel player in the control of MGMT expression and temozolomide resistance of glioblastoma cancer stem cells. We showed that inhibition of JNK, either pharmacologically or by RNA interference, in stem-like glioblastoma cells derived directly from glioblastoma tissues reduces their MGMT expression and temozolomide resistance. Importantly, sensitization of stem-like glioblastoma cells to temozolomide by JNK inhibition was dependent on MGMT expression, implying that JNK controls temozolomide resistance of stem-like glioblastoma cells through MGMT expression. Our findings suggest that concurrent use of JNK inhibitors with temozolomide may be a rational therapeutic approach to effectively target the cancer stem cell population in the treatment of glioblastoma.
\end{abstract}

Correspondence to: Professor Chifumi Kitanaka, Department of Molecular Cancer Science, Yamagata University School of Medicine, Yamagata 990-9585, Japan

E-mail: ckitanak@med.id.yamagata-u.ac.jp

Key words: glioma-initiating cell, cancer stem cell, combination chemotherapy, chemoresistance, JNK, $\mathrm{O}^{6}$-methylguanine DNA methyltransferase

\section{Introduction}

Glioblastoma multiforme, the most common primary brain tumor in adults, is one of the deadliest of all human cancers with a median survival of $<2$ years at best, even with the current standard of care consisting of maximal surgical resection followed by radiotherapy with concomitant and adjuvant temozolomide $(1,2)$. Early recurrence, which eventually becomes fatal, is inevitable even after successful initial treatment of glioblastoma, for which cancer stem cells are now deemed responsible $(3,4)$. Cancer stem cells are a small subpopulation of cancer cells comprising a tumor that possess stem cell-like properties as well as the capacity to initiate a tumor and are often associated with resistance against conventional chemo- and radiotherapies $(5,6)$. Elimination of the cancer stem cell population by overcoming their therapy resistance, therefore, is considered a key to successful management of glioblastoma as well as to realization of long-term survival of patients with this devastating disease. Although it still remains an open question whether or not cancer stem cells of glioblastoma are invariably resistant to temozolomide, current literature unanimously indicates that they often express $\mathrm{O}^{6}$-methylguanine DNA methyltransferase (MGMT) and that MGMT expression is associated with high resistance to temozolomide (7). MGMT is a repair enzyme that rapidly removes the methyl group attached by temozolomide at the $\mathrm{O}^{6}$ position of the guanine residue. As such, MGMT expression is considered to be a major mechanism for temozolomide resistance of glioblastoma, which has indeed been supported by a wealth of data from both clinical and basic studies (8-12). Thus, the accumulating evidence supports the idea that elucidating how MGMT expression is regulated in cancer stem cells of glioblastoma would be a valuable strategy to control its expression and, by so doing, render them susceptible to temozolomide treatment.

In the present study, we dissected the molecular mechanism of temozolomide resistance using glioblastoma cells having cancer stem cell properties (stem-like glioblastoma 
cells) that were derived directly from glioblastoma patients. We found that the JNK pathway is critically involved in the temozolomide resistance and MGMT expression of stem-like glioblastoma cells that express MGMT. JNK inhibition failed to sensitize stem-like glioblastoma cells without MGMT expression, suggesting that JNK may contribute to temozolomide resistance of stem-like glioblastoma cells in an MGMT expression-dependent manner.

\section{Materials and methods}

Reagents and antibodies. SP600125 and temozolomide were purchased from Calbiochem (La Jolla, CA, USA) and LKT Laboratories, Inc. (St. Paul, MN, USA), respectively, and were dissolved in dimethylsulfoxide (DMSO) to prepare $50 \mathrm{mM}$ stock solutions. Anti-c-Jun (no. 9165) and anti-phospho-c-Jun (Ser 63) (no. 9261) antibodies were purchased from Cell Signaling Technology, Inc. (Beverly, MA, USA). Anti- $\beta$-actin (A1978) was from Sigma (St. Louis, MO, USA). Anti-JNK1 (sc-474) and anti-JNK2 (sc-7345) were from Santa Cruz Biotechnology, Inc. (Santa Cruz, CA, USA). Anti-MGMT (ab39253) was purchased from Abcam (Cambridge, UK).

Cell culture. Patient-derived stem-like glioblastoma cells used in this study (GS-Y01, GS-Y03, GS-NCC01 and TGS01) were directly established from glioblastoma tissues in accordance with protocols approved by the Institutional Review Boards of the institutions where they were established. The establishment and characterization of the stem-like glioblastoma cells have been described (13-15). The cells were maintained under the monolayer stem cell culture condition reported previously $(14,15)$.

Temozolomide treatment and liveldead cell assays. Throughout this study, for temozolomide treatment, cells were exposed to $50 \mu \mathrm{M}$ temozolomide for $4 \mathrm{~h}$, after which they were cultured in the absence of temozolomide until being subjected to analyses. In each set of treatment, cells were examined for their viability at the end of pretreatment (i.e., SP600125 treatment and/or knockdown of JNK and/or MGMT), and an equal number of viable cells were used for subsequent temozolomide/control treatment. Live and dead cells were identified by their ability and inability to exclude vital dyes [trypan blue and propidium iodide (PI)], respectively. Unless otherwise specified, both adherent and non-adherent cells in the dishes were collected, and after centrifugation, re-suspended in phosphate-buffered saline (PBS). The cell suspension was then mixed with an equal volume of PBS containing trypan blue $(0.4 \% \mathrm{w} / \mathrm{v}$, final concentration, $0.2 \%)$ and examined under a phase-contrast microscope using a hemocytometer. The percentage of dead cells was defined as $100 \mathrm{x}$ the number of dead cells / (the number of viable cells + the number of dead cells). Alternatively, cells were incubated in situ with PI $(1 \mu \mathrm{g} / \mathrm{ml})$ and Hoechst $33342(10 \mu \mathrm{g} / \mathrm{ml})$ for $10 \mathrm{~min}$ at $37^{\circ} \mathrm{C}$ in the $\mathrm{CO}_{2}$ incubator, to stain dead cells and the cell nuclei, respectively. Then the numbers of PI- and Hoechst-positive cells were scored under a fluorescence microscope (CKX41; Olympus, Tokyo, Japan), and the percentage of PI-positive cells (dead cells) against Hoechst-positive cells (total cells) was determined.
Colony formation assay. For colony formation, cells were seeded at a low, colony-forming density $\left(5 \times 10^{3}\right.$ cells per $60-\mathrm{mm}$ dish) and cultured for $\sim 4$ weeks. The cells were then fixed with formaldehyde $(4 \% \mathrm{v} / \mathrm{v})$ followed by staining with crystal violet $(0.1 \% \mathrm{w} / \mathrm{v})$. Colonies [consisting of 8 or more cells (progenies) derived from a single cell] were counted using a microscope.

Gene silencing by siRNA. siRNAs against human JNK1 (HSS108547), JNK2 (HSS108550), MGMT (HSS106519) and Stealth RNAi ${ }^{\mathrm{TM}}$ siRNA negative control duplexes (Medium GC Duplexes no. 2) were purchased from Invitrogen Life Technologies (Carlsbad, CA, USA). Transfection of siRNAs was performed using Lipofectamine RNAiMAX (Invitrogen Life Technologies) according to the manufacturer's instructions.

Immunoblot analysis. Cells were washed with ice-cold PBS and lysed in the RIPA buffer [10 mM Tris-HCl ( $\mathrm{pH} 7.4)$, $0.1 \%$ SDS, $1 \%$ sodium deoxycholate, $150 \mathrm{mM} \mathrm{NaCl}, 1 \mathrm{mM}$ EDTA, $1.5 \mathrm{mM} \mathrm{Na} \mathrm{VO}_{4}, 10 \mathrm{mM} \mathrm{NaF}, 10 \mathrm{mM}$ sodium pyrophosphate, $10 \mathrm{mM}$ sodium $\beta$-glycerophosphate and $1 \%$ protease inhibitor cocktail set III (Calbiochem)]. After centrifugation for $10 \mathrm{~min}$ at $14,000 \mathrm{xg}$ at $4^{\circ} \mathrm{C}$, the supernatants were recovered as the cell lysates, and the protein concentration of the cell lysates was determined by the BCA protein assay kit (Pierce Biotechnology, Inc., Rockford, IL, USA). Cell lysates containing equal amounts of protein were separated by SDS-PAGE and transferred to a polyvinylidene difluoride membrane. The membrane was probed with a primary antibody and then with an appropriate HRP-conjugated secondary antibody according to the protocol recommended by the manufacturer of each antibody. Immunoreactive bands were visualized using Immobilon Western Chemiluminescent HRP Substrate (Millipore, Billerica, MA, USA).

$R T-P C R$. Total RNA was extracted with TRIzol (Invitrogen Life Technologies). Total RNA was reverse-transcribed into cDNA using PrimeScript ${ }^{\mathrm{TM}}$ 1st strand cDNA Synthesis kit (Takara, Tokyo, Japan) according to the manufacturer's instructions. Amplification was performed with cycles of $97^{\circ} \mathrm{C}$ for $30 \mathrm{sec}, 55^{\circ} \mathrm{C}$ for $30 \mathrm{sec}$, and $72^{\circ} \mathrm{C}$ for $1 \mathrm{~min}$ in a thermal cycler (Takara PCR Thermal Cycler Dice). PCR cycles were 30 for MGMT and 25 for $\beta$-actin. RT-PCR analysis was performed with the following primers: MGMT (forward: 5'-GCTGGAGCTGTCTGGTTGTGAG, reverse: 5'-GCGC GGCTTTGGGGTTGC), $\beta$-actin (forward: 5'-CCCATGCCA TCCTGCGTCTG, reverse: 5'-CGTCATACTCCTGCTTG CTG).

Statistical analysis. Results are expressed as the means \pm SD, and differences were compared using the 2-tailed Student's t-test. P-values $<0.05$ were considered statistically significant and indicated with asterisks in the figures.

\section{Results}

SP600125, a JNK inhibitor, sensitizes stem-like glioblastoma cells with MGMT expression to temozolomide. We have previously demonstrated that the JNK pathway is commonly activated in stem-like glioblastoma cells compared to their 
A

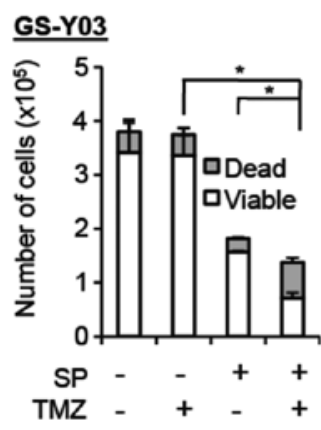

C
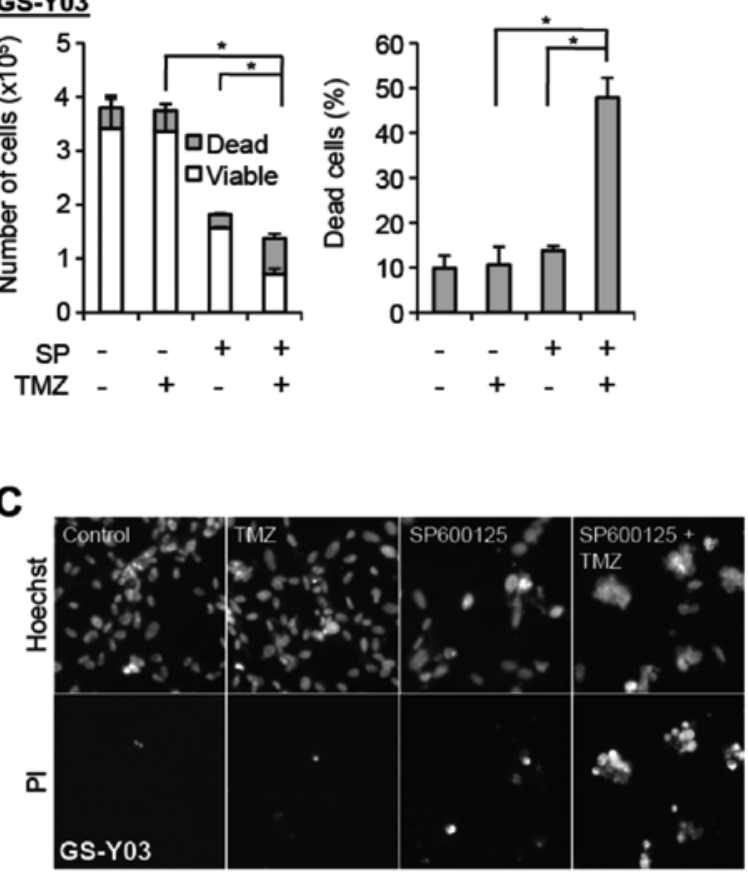

B
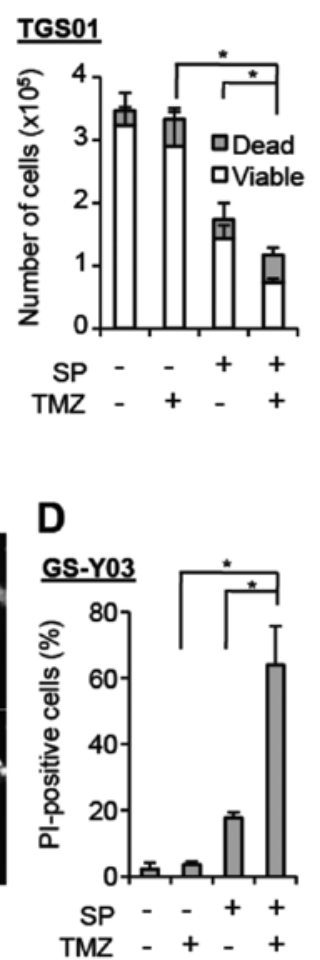
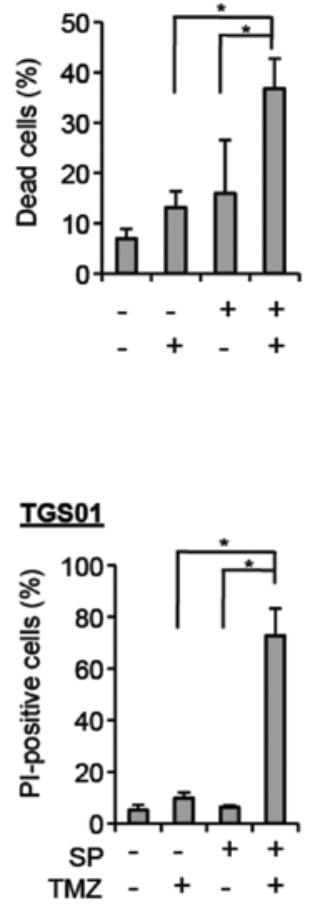
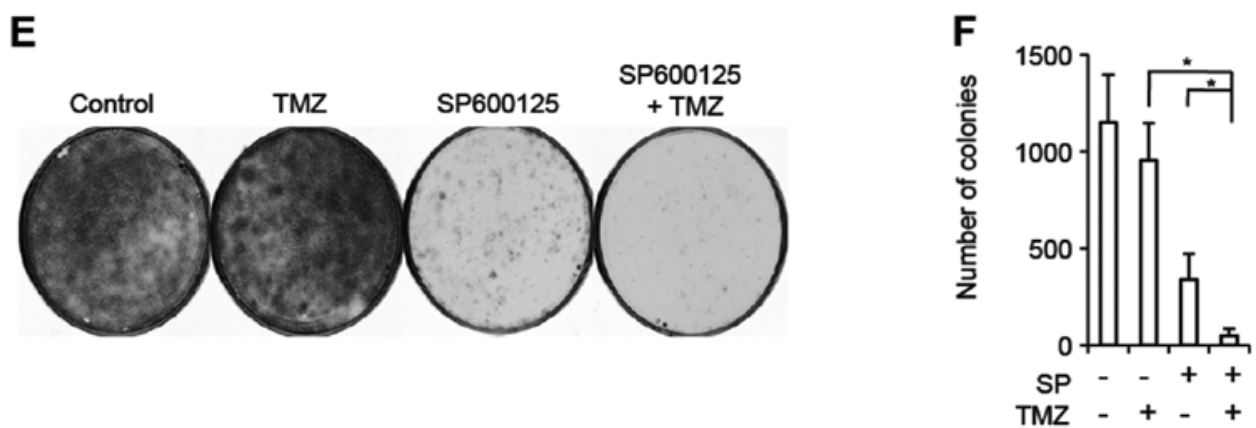

Figure 1. SP600125 sensitizes stem-like glioblastoma cells with MGMT expression to temozolomide. (A-D) GS-Y03 and TGS01 stem-like glioblastoma cells pretreated with or without SP600125 (SP, $20 \mu \mathrm{M}$ for GS-Y03, $30 \mu \mathrm{M}$ for TGS01) for 3 days were subsequently treated with or without temozolomide (TMZ, $50 \mu \mathrm{M}$ ) for $4 \mathrm{~h}$ and then cultured in the absence of TMZ for 3 days. Then the numbers of viable and dead cells (left panel) as well as the percentage of dead cells (right panel) were determined for GS-Y03 cells (A) and TGS01 cells (B). Alternatively, the cells were subjected to cell death analysis using propidium iodide (PI). Representative fluorescence images of Hoechst- (upper rows) and PI- (lower rows) positive cells (C), as well as the percentage of PI-positive cells (dead cells) relative to Hoechst-positive cells (total cells) (D) are shown. (E and F) GS-Y03 cells pretreated with or without SP600125 (20 $\mu$ M) for 3 days were subjected to colony formation assay with or without temozolomide treatment (TMZ, $50 \mu \mathrm{M}$ for $4 \mathrm{~h}$ ). An image of a representative experiment (E) and the number of colonies (F) are presented. In (A), (B), (D) and (F), the values in the graphs represent means \pm standard deviations of three independent experiments. ${ }^{*} \mathrm{P}<0.05$ [note that the numbers of viable cells are compared in the left panels of (A) and (B)].

differentiated counterparts and that the JNK activity is required for the maintenance of the stemness/tumor-initiating capacity of stem-like glioblastoma cells (14). Since cancer stem cells are in general associated with chemo- and radio-resistance (6), we wondered if JNK also has a role in determining their sensitivity/resistance against temozolomide, the current chemotherapeutic agent of choice in the treatment of glioblastoma (16). To address this question, we used stem-like glioblastoma cells derived directly from glioblastoma patients and asked whether the cytotoxic effect of temozolomide on these stemlike glioblastoma cells would be modulated by treatment with SP600125, a chemical inhibitor of JNK. We first tested the idea using GS-Y03 stem-like glioblastoma cells, which express MGMT and are virtually refractory to clinically relevant concentrations $(\sim 50 \mu \mathrm{M})$ of temozolomide (Fig. 1A and C-F; see also Fig. 3). When GS-Y03 cells pretreated with SP600125 were exposed to temozolomide at $50 \mu \mathrm{M}$ and collected 3 days after the temozolomide treatment to determine their viability, we found that SP600125 pretreatment significantly increased the proportion of dead cells after temozolomide treatment, while SP600125 pretreatment alone caused only marginal increase in the proportion of dead cells (Fig. 1A). Since fragile dead cells could be lost during the cell collection procedure, we also stained dead cells in situ with a fluorescent vital dye 
A

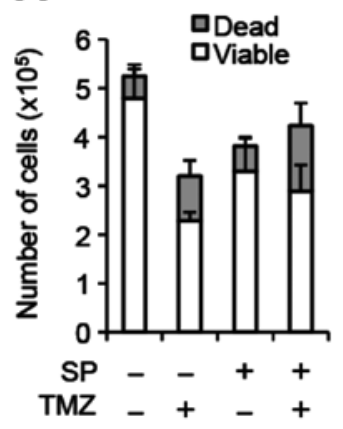

B

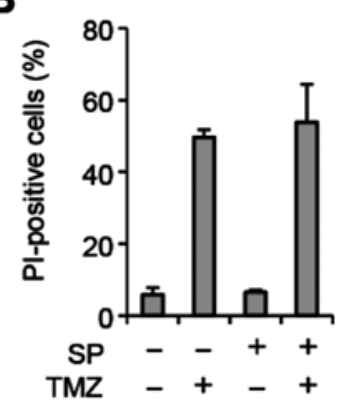

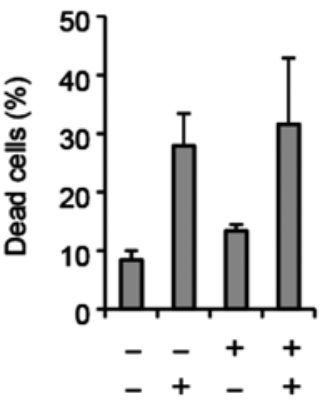

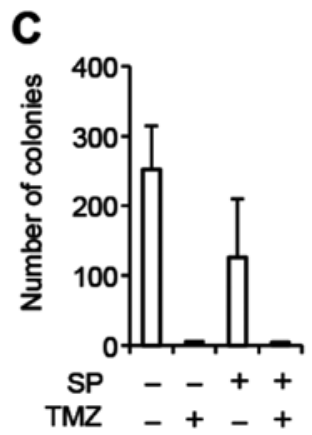

Figure 2. Failure of SP600125 to promote temozolomide-induced cell death in stem-like glioblastoma cells lacking MGMT expression (GS-NCC01 cells). (A and B) GS-NCC01 stem-like glioblastoma cells pretreated with or without SP600125 (SP, $20 \mu \mathrm{M})$ for 3 days were subsequently treated with or without temozolomide (TMZ, $50 \mu \mathrm{M}$ ) for $4 \mathrm{~h}$ and then cultured in the absence of TMZ for 3 days. Then, the numbers of viable and dead cells (left panel) as well as the percentage of dead cells (right panel) were determined. Alternatively, the cells were subjected to cell death analysis using propidium iodide (PI). The percentage of PI-positive cells (dead cells) relative to Hoechst-positive cells (total cells) (B) are shown. (C) GS-NCC01 cells pretreated with or without SP600125 (SP, $20 \mu \mathrm{M})$ for 3 days were subjected to colony formation assay with or without temozolomide treatment (TMZ, $50 \mu \mathrm{M}$ for $4 \mathrm{~h}$ ), and the number of colonies was determined. In (A-C), the values in the graphs represent means \pm standard deviations of three independent experiments.

Hoechst 33342. Although it turned out that SP600125 pretreatment alone somewhat increased cell death when examined by this method, cell death was further increased by exposing cells to temozolomide after SP600125 pretreatment, whereas temozolomide alone had no cell death-increasing effect on GS-Y03 cells (Fig. 1C and D). Thus, the results of the two cell death assays consistently suggested that SP600125 renders GS-Y03 cells, which are otherwise highly resistant to temozolomide, sensitive to the chemotherapeutic agent. We then wished to determine whether SP600125 actually augments the suppressive effect of temozolomide on clonogenic survival, instead of simply changing the time kinetics of cell death, i.e., just letting cell death occur at earlier time-points that would otherwise occur at later time-points. To address this question, we conducted the colony formation assay. SP600125 pretreatment of GS-Y03 cells alone efficiently inhibited colony formation, most likely because SP600125 caused a proliferation block associated with differentiation. However, colony formation was further inhibited when GS-Y03 cells were exposed to temozolomide after SP600125 pretreatment, whereas temozolomide alone had no discernible inhibitory effect on

colony formation (Fig. 1E and F). The results of the colony formation assay thus indicated that SP600125 efficiently suppresses clonogenic survival of stem-like glioblastoma cells in combination with temozolomide. Intriguing enough, whereas we obtained essentially similar results (i.e., sensitization to temozolomide by SP600125 pretreatment) from TGS01 and GS-Y01 cells that express MGMT $(17,18)$ (Fig. 1B and D; data not shown for GS-Y01), we observed no such effect of SP600125 on another patient-derived stem-like glioblastoma cell line GS-NCC01, which do not express MGMT (Fig. 2; Fig. 3A and F). In GS-NCC01 cells, temozolomide exposure alone caused substantial cell death consistent with the lack of MGMT expression, and SP600125 pretreatment failed to increase cell death either alone or in combination with temozolomide (Fig. 2A and B). In line with the results of the cell death assay, temozolomide exposure alone almost totally eliminated colony formation, and we saw no further decrease in colony formation by SP600125 pretreatment (Fig. 2C). Together, these results suggest that SP600125 may specifically sensitize MGMT-expressing, temozolomide-resistant stemlike glioblastoma cells to temozolomide treatment.

Inhibition of MGMT expression in stem-like glioblastoma cells by SP600125. Since SP600125 specifically sensitized stem-like glioblastoma cells with MGMT expression to temozolomide, we next asked whether SP600125 did so through modulation of the expression and/or activity of MGMT. To this end, we first examined the effect of SP600125 on the mRNA expression of MGMT in stem-like glioblastoma cells. The results indicated that GS-Y01, GS-Y03 and TGS01 but not GS-NCC01 cells express detectable levels of MGMT mRNA, which were significantly decreased after 3-day pretreatment with SP600125 (Fig. 3A). The time course analysis using GS-Y03 cells revealed that MGMT mRNA gradually declined over 3 days during the SP600125 pretreatment period (Fig. 3B). Consistent with the change of the mRNA level, the SP600125 pretreatment also decreased the expression of the MGMT protein, with some time lag compared to that of mRNA (Fig. 3C). Further analysis showed that SP600125 inhibited both JNK activity (as monitored by phospho-c-Jun expression) and MGMT protein expression at $10 \mu \mathrm{M}$ or higher concentrations (Fig. 3D). Inhibition of MGMT protein expression by SP600125 was similarly demonstrated in TGS01 and GS-Y01 cells (Fig. 3E; data not shown for GS-Y01). We also confirmed that GS-NCC01 cells do not express MGMT protein and that SP600125 does inhibit the JNK activity in GS-NCC01 cells, excluding the possibility that the lack of sensitization effect is due to failure of SP600125 to inhibit JNK in GS-NCC01 cells (Fig. 3F).

JNK knockdown inhibits MGMT expression and sensitizes stem-like glioblastoma cells to temozolomide. To definitively establish that JNK is actually involved in the regulation of the MGMT expression and temozolomide resistance of stem-like glioblastoma cells, we knocked down JNK and examined its effect on these parameters. To achieve effective reduction in the JNK activity, we introduced into GS-Y03 and TGS01 cells a combination of siRNAs against JNK1 and JNK2. Successful knockdown of JNK1 and JNK2 resulted in reduced expression of phospho-c-Jun, and also caused decreased MGMT 
A

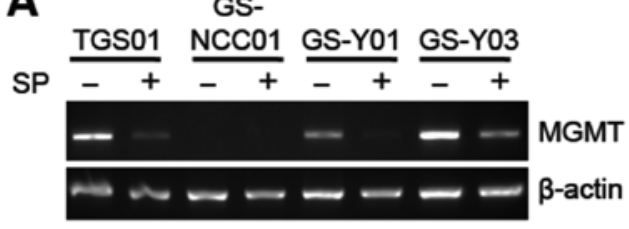

B

\begin{tabular}{|c|c|c|c|c|}
\hline$(-)$ & \multicolumn{3}{|c|}{ SP600125 } & \\
\hline 3 & 1 & 2 & 3 & (days) \\
\hline 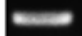 & $=$ & $=$ & - & MGMT \\
\hline & & & & \\
\hline
\end{tabular}

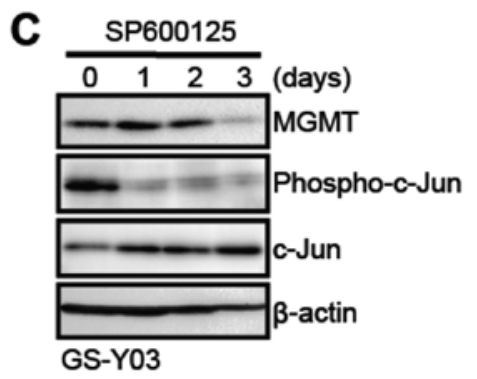

E

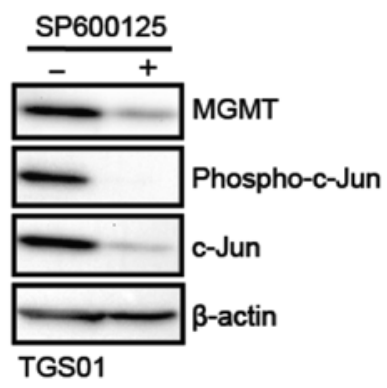

D

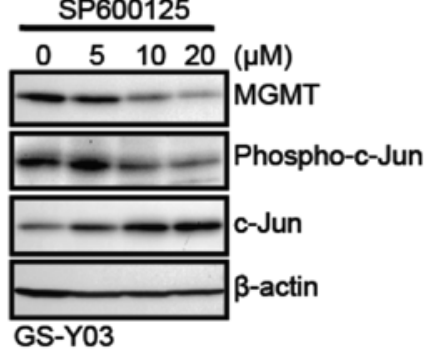

$\mathbf{F}$

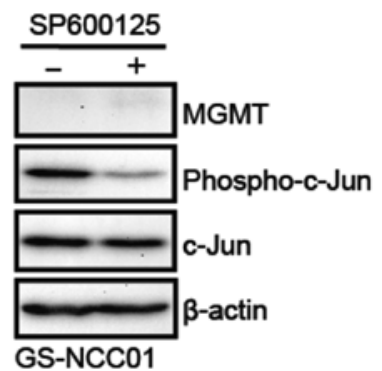

Figure 3. SP600125 inhibits MGMT expression in stem-like glioblastoma cells. (A) The indicated stem-like glioblastoma cells treated with or without SP600125 (SP, $30 \mu \mathrm{M}$ for TGS01, $20 \mu \mathrm{M}$ for the others) for 3 days were subjected to RT-PCR analysis of MGMT mRNA expression. (B) GS-Y03 cells treated with or without SP600125 $(20 \mu \mathrm{M})$ for the indicated time periods were subjected to RT-PCR analysis of MGMT mRNA expression. (C) GS-Y03 cells treated without (0 day) or with SP600125 (20 $\mu \mathrm{M})$ for the indicated time periods (1-3 days) were subjected to immunoblot analysis of the indicated proteins. (D) GS-Y03 cells treated with the indicated concentrations of SP600125 for 3 days were subjected to immunoblot analysis of the indicated proteins. (E and F) TGS01 (E) and GS-NCC01 (F) cells treated with SP600125 (30 $\mu \mathrm{M}$ for TGS01, $20 \mu \mathrm{M}$ for GS-NCC01) for 3 days were subjected to immunoblot analysis of the indicated proteins.

expression in parallel with the reduced JNK activity (Fig. 4A). The stem-like glioblastoma cells were then examined for their sensitivity to temozolomide in the presence and absence of JNK knockdown. Whereas temozolomide exposure alone in the absence of JNK knockdown did not have a significant impact on cell viability, the proportion of dead cells was substantially increased when cells were exposed to temozolomide after JNK knockdown (Fig. 4B-E). In line with the results of the cell death assay, JNK knockdown cells formed apparently less colonies after temozolomide exposure in the colony formation assay, in contrast to the control cells which formed colonies quite efficiently irrespective of temozolomide exposure (Fig. 4F and G). In conjunction with the results of the analyses using SP600125, these results demonstrate that JNK plays a critical role in the maintenance of the MGMT expression and temozolomide resistance of stem-like glioblastoma cells.

MGMT-dependent contribution of JNK to temozolomide resistance of stem-like glioblastoma cells. So far, we have demonstrated that JNK plays a role in the maintenance of the MGMT expression and temozolomide resistance of stemlike glioblastoma cells that express MGMT. We then wished to ask whether downregulation of MGMT expression is the mechanism for the JNK inhibition-mediated sensitization of stem-like glioblastoma cells to temozolomide. To determine whether the sensitization effect of JNK inhibition is dependent on MGMT expression, we examined the impact of MGMT knockdown on the temozolomide-sensitizing effect of JNK inhibition. In the control experiment, GS-Y03 cells transfected with a control siRNA were treated with or without temozolomide in the absence or presence of SP600125 pretreatment. Consistent with the earlier results, under this experimental condition, SP600125 inhibited MGMT expression and sensitized the cells to temozolomide (Fig. 5A and B). In sharp contrast, when the cells were transfected with a siRNA against MGMT to knockdown MGMT expression, the cells became sensitive to temozolomide alone (compare siMGMT and siMGMT+TMZ) and the temozolomide-sensitizing effect of SP600125 pretreatment was lost under this condition (compare siMGMT+TMZ and siMGMT+SP+TMZ) (Fig. 5A and B). Unexpectedly, SP600125 pretreatment promoted cell death when combined with MGMT knockdown, the reason for which remains currently unknown. Nevertheless, the observation that SP600125 pretreatment no longer promoted temozolomide-induced cell death in MGMT knockdown 
A

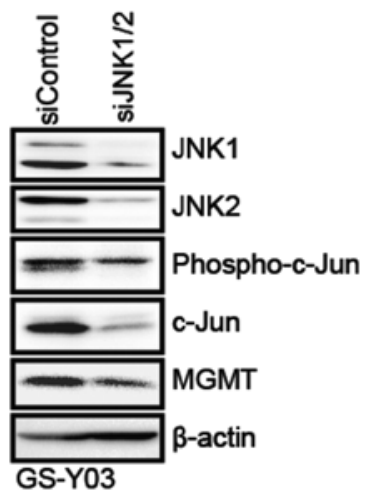

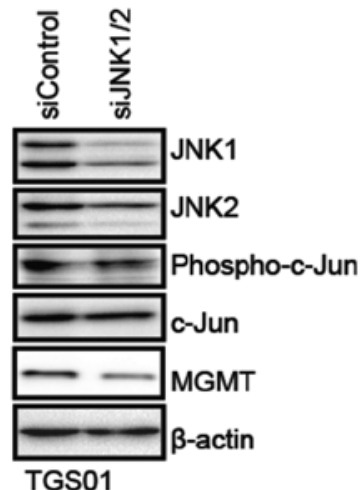

C $\underline{\text { TGS01 }}$

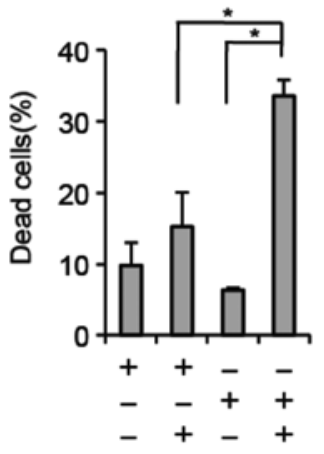

B

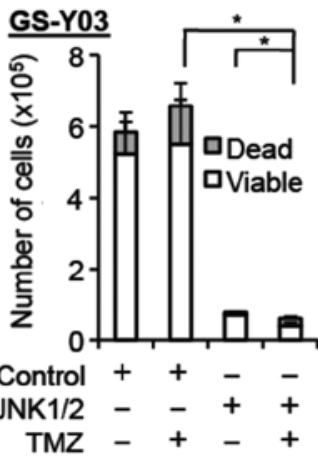

D

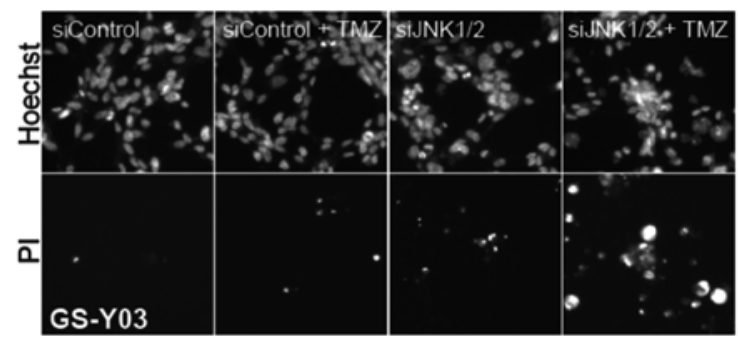

E $\underline{\text { GS-Y03 }}$
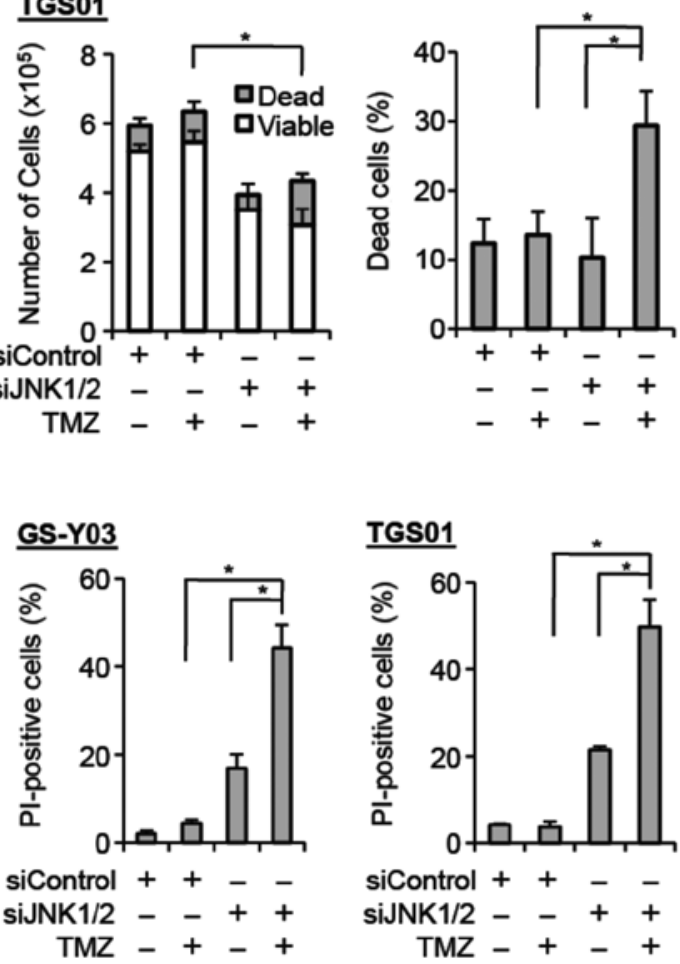

$\mathbf{F}$

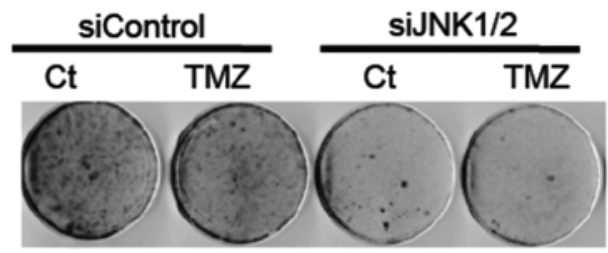

G

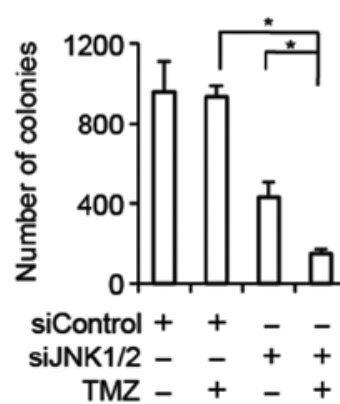

Figure 4. JNK knockdown inhibits MGMT expression and sensitizes stem-like glioblastoma cells to temozolomide. (A) The indicated stem-like glioblastoma cells were transiently transfected with a combination of siRNAs against JNK1 and JNK2 (siJNK1/2) or with a control siRNA (siControl). After 4 days, the transfected cells were subjected to immunoblot analysis for the expression of the indicated proteins. (B-E) The indicated stem-like glioblastoma cells transiently transfected with the indicated siRNAs for 4 days were subsequently treated with or without temozolomide (TMZ, $50 \mu \mathrm{M})$ for $4 \mathrm{~h}$ and cultured in the absence of TMZ for 3 days thereafter. Then, the numbers of viable and dead cells (left panel) as well as the percentage of dead cells (right panel) were determined for GS-Y03 cells (B) and TGS01 cells (C). Alternatively, the cells were subjected to cell death analysis using propidium iodide (PI). Representative fluorescence images of Hoechst- (upper rows) and PI- (lower rows) positive cells (D), as well as the percentage of PI-positive cells (dead cells) relative to Hoechst-positive cells (total cells) (E) are shown. (F and G) GS-Y03 cells transiently transfected with a combination of siRNAs against JNK1 and JNK2 (siJNK1/2) or with a control siRNA (siControl) for 4 days were then subjected to colony formation assay with or without temozolomide treatment (TMZ, $50 \mu \mathrm{M}$ for $4 \mathrm{~h}$ ). An image of a representative experiment (F) and the number of colonies (G) are presented. In (B), (C), (E), and (G), the values in the graphs represent means \pm standard deviations of three independent experiments. "P<0.05 [note that the numbers of viable cells are compared in the left panels of (B) and (C)]. 
A

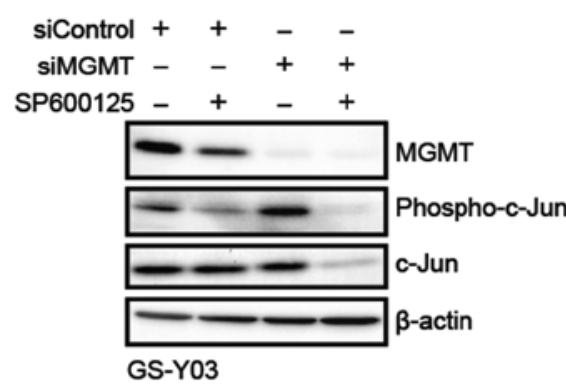

C

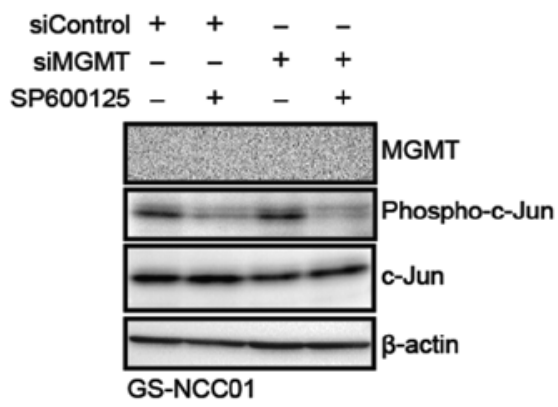

B
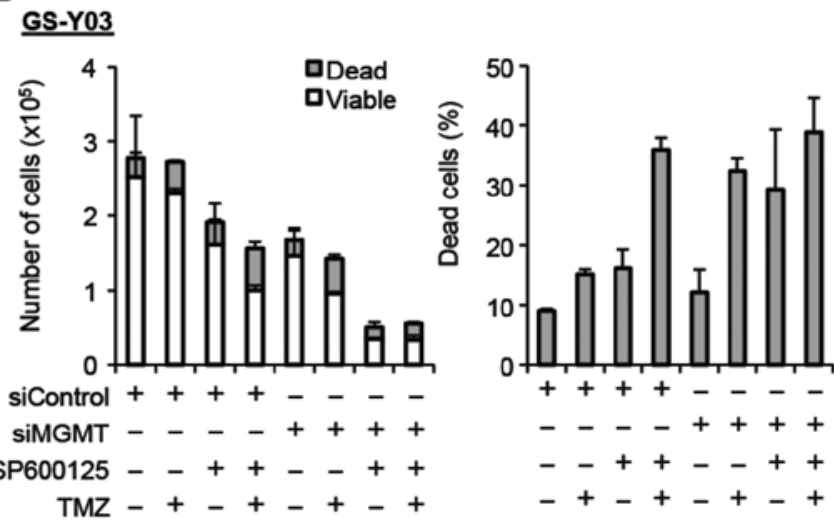

D

\section{GS-NCC01}

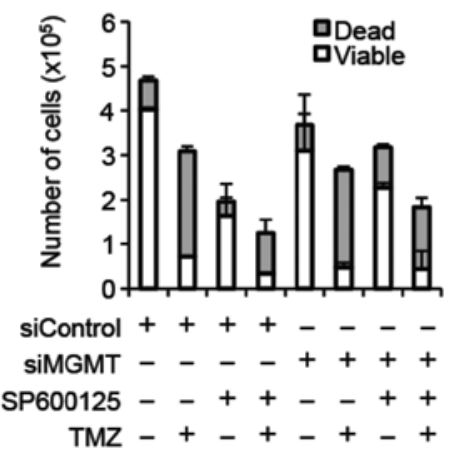

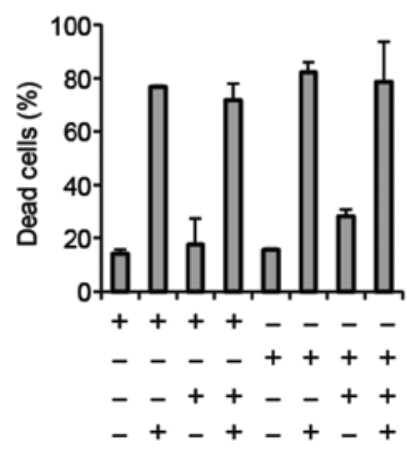

Figure 5. MGMT expression-dependent sensitization of stem-like glioblastoma cells to temozolomide by SP600125. (A) GS-Y03 cells transiently transfected with either a siRNA against MGMT (siMGMT) or a control siRNA (siControl) for $24 \mathrm{~h}$ were treated with or without $20 \mu \mathrm{M}$ SP600125 for 3 days and then subjected to immunoblot analysis of the indicated proteins. (B) GS-Y03 cells transiently transfected with either a siRNA against MGMT (siMGMT) or a control siRNA (siControl) for $24 \mathrm{~h}$ were treated with or without SP600125 $(20 \mu \mathrm{M})$ for 3 days. The cells were then further treated with or without temozolomide (TMZ, $50 \mu \mathrm{M}$ ) for $4 \mathrm{~h}$ and cultured in the absence of TMZ thereafter. The numbers of viable and dead cells (left panel) as well as the percentage of dead cells (right panel) were determined 3 days after the TMZ treatment. (C) GS-NCC01 cells transiently transfected with the indicated siRNAs for $24 \mathrm{~h}$ were treated with or without SP600125 $(20 \mu \mathrm{M})$ for 3 days and then subjected to immunoblot analysis of the indicated proteins. (D) GS-NCC01 cells transiently transfected with the indicated siRNAs for $24 \mathrm{~h}$ were treated with or without SP600125 $(20 \mu \mathrm{M})$ for 3 days. The cells were then further treated with or without temozolomide (TMZ, $50 \mu \mathrm{M}$ ) for $4 \mathrm{~h}$ and cultured in the absence of TMZ thereafter. The numbers of viable and dead cells (left panel) as well as the percentage of dead cells (right panel) were determined 3 days after the TMZ treatment. In (B) and (D), the values in the graphs represent means \pm standard deviations of three independent experiments.

cells supports the idea that the temozolomide-sensitizing effect of JNK inhibition is lost upon MGMT knockdown. Finally, to rule out the possibility that the siRNA against MGMT cancelled the sensitizing effect of SP600125 in an MGMT-independent manner, we conducted a similar experiment using GS-NCC01 cells that do not express MGMT. The results indicated GS-NCC01 cells remained equally sensitive to temozolomide either in the absence or presence of SP600125 pretreatment irrespective of whether or not MGMT was knocked down (Fig. 5C and D). Collectively, these findings suggest that regulation of MGMT expression is a mechanism by which JNK contributes to temozolomide resistance of stem-like glioblastoma cells.

\section{Discussion}

Cancer stem cells are now increasingly recognized as a critical therapeutic target in cancer treatment. Since cancer stem cells are often associated with inherent resistance against conventional chemo- and radio-therapies, overcoming their therapy resistance is among the most important issues in cancer therapeutics $(3,4)$. In the case of glioblastoma, cancer stem cells are frequently, albeit not always, resistant to temozolomide, the standard chemotherapeutic agent for the treatment of glioblastoma (7). Accumulating evidence indicates that temozolomide-resistant glioblastoma cancer stem cells are associated with increased MGMT expression (8-12), and we and others have indeed demonstrated that MGMT expression is responsible for the temozolomide resistance of glioblastoma cancer stem cells $(12,18)$. Thus, the evidence suggests that therapies targeting MGMT expression/activity in combination with temozolomide would be a rational strategy to treat temozolomide-resistant glioblastoma cancer stem cells, and also underscores the necessity of dissecting the mechanism of MGMT regulation in glioblastoma cancer stem cells. In this regard, we have previously demonstrated that the MAPK pathway regulates MGMT expression in stem-like glioblastoma cells through the MDM2-p53 axis (18). Only recently, the BMP2-HIF1- $\alpha$, mGlu3-PI3K/NF- $\kappa$ B, and ZEB1-c-MYB pathways have been implicated in the regulation of MGMT 
expression in glioma stem cells (19-21). While these pathways are expected to become attractive candidates for therapeutic targeting to overcome temozolomide resistance of glioblastoma cancer stem cells, apparently, the information is still limited and much remains unknown regarding the MGMT regulation in glioblastoma cancer stem cells.

Herein, we have provided lines of evidence that JNK is involved in the maintenance of MGMT expression and temozolomide resistance of stem-like glioblastoma cells. Both pharmacological and genetic inhibition of JNK resulted in decreased MGMT expression and reduced temozolomide resistance of otherwise temozolomide-resistant stem-like glioblastoma cells with MGMT expression. Significantly, JNK inhibition-mediated sensitization of stem-like glioblastoma cells to temozolomide did not occur in cells without MGMT expression (either originally lacking MGMT expression or after MGMT knockdown). Thus, to our knowledge, this is the first study to demonstrate the critical role of the JNK pathway in MGMT-dependent temozolomide resistance of stem-like glioblastoma cells. Intriguingly, an earlier report showed that SP600125 sensitized serum-cultured U87 cells to temozolomide (22). Given that U87 is a human glioblastoma cell line widely known to be sensitive to temozolomide due to lack of MGMT expression (23-25), the mechanism for the temozolomide-sensitizing effect of SP600125 in that study was most likely MGMT-independent and therefore distinct from the one proposed in the present study. Moreover, since JNK inhibition in the study was done solely by use of SP600125 known to have well-characterized off-target effects (26-28), it could even be possible that the sensitizing effect of SP600125 in U87 cells was JNK-independent. It needs to be emphasized here, however, that the results of our study do not necessarily exclude the possibility that JNK could also contribute to temozolomide resistance independently of MGMT.

We have demonstrated in this study that JNK plays a role in the control of MGMT expression in stem-like glioblastoma cells, but the molecular pathway connecting JNK to MGMT expression remains to be determined. Although MGMT could be regulated at the protein level as exemplified by STAT3-mediated regulation of MGMT (29), our data clearly indicate that JNK regulation of MGMT expression occurs at the mRNA level, suggesting that JNK likely contributes to MGMT expression through promotion of its transcription. To date, a number of transcription factors and complexes, such as Sp1, AP-1, c-Myc, HIF1- $\alpha$, c-Myb, and NF- $\kappa B$, have been reported to directly bind and activate the MGMT gene promoter (20,21,30-33). Among these factors and complexes, Sp1 and c-Myc, let alone AP-1 which is a jun/fos heterodimer, may be of particular interest in that they are direct targets of JNK-mediated phosphorylation and activation. Earlier reports indicated that JNK maintains the stability of the Sp1 protein through phosphorylation at Thr278/739 $(34,35)$. Another report demonstrated that JNK enhances the pro-apoptotic activity of c-Myc through phosphorylation at Ser62/71 (36). Whether and how JNK is connected with those and other transcription factors/complexes, either directly or indirectly, is an intriguing issue that still needs to be addressed.

While future studies are expected to shed light on the detailed molecular mechanism underlying JNK-mediated control of MGMT expression and temozolomide resistance in stem-like glioblastoma cells, JNK would nonetheless remain superior as a therapeutic target to any of the possible targets that would be newly identified in future studies, because JNK is a key molecule that stands at the 'crossroads' of the molecular pathways governing stemness and chemoresistance. We have previously demonstrated that JNK plays an essential role in the maintenance of the stem cell properties and tumor-initiating capacity of stem-like glioblastoma cells and that systemic administration of SP600125 as a monotherapy effectively deprives their self-renewal and tumor-initiating capacities in vivo (14). As such, the data of our previous study alone would have suggested use of JNK inhibitor as a maintenance monotherapy after the completion of the initial chemoradiotherapy, for the purpose of targeting glioblastoma cancer stem cells that have evaded chemoradiotherapy. However, now that we have demonstrated that a JNK inhibitor inhibited the temozolomide resistance of glioblastoma cancer stem cells along with their stemness, there is a rationale for concomitant use of a JNK inhibitor and temozolomide for the treatment of glioblastoma. Such a combinational use of a JNK inhibitor and temozolomide is also of value in another perspective, because temozolomide would kill and irreversibly eliminate glioblastoma cancer stem cells that have undergone differentiation and lost the capacity to initiate tumors as a result of JNK inhibitor treatment but may not necessarily be without the risk of recovering their stem cell properties and tumor-initiating capacity (37). Thus, JNK is a highly beneficial molecular target to control the stemness and chemoresistance of glioblastoma cancer stem cells at the same time and therefore will remain an attractive therapeutic target to treat glioblastoma cancer stem cells.

In conclusion, we have demonstrated in this study that JNK contributes to the MGMT expression and temozolomide resistance of stem-like glioblastoma cells. Together with the known critical role of JNK in the maintenance of the stemness of stem-like glioblastoma cells $(14,38)$, our current findings provide a molecular rationale for combining JNK inhibitors with temozolomide to effectively target glioblastoma stem cells. The therapeutic significance of such combination treatment will be tested in future preclinical and clinical studies.

\section{Acknowledgements}

We thank Drs Tomoki Todo and Nobuhito Saito at the University of Tokyo for generously providing us with the TGS01 cells and Dr Tomoko Kagawa for her continuous support/encouragement. This study was supported by Grantsin-Aid for Scientific Research, for Challenging Exploratory Research, and for Young Scientists from the Ministry of Education, Culture, Sports, Science and Technology of Japan, by a Grant-in-Aid from the Global COE Program of the Japan Society for the Promotion of Science, by the National Cancer Center Research and Development Fund (23-A-20), and by a grant from the Japan Brain Foundation.

\section{References}

1. Wen PY and Kesari S: Malignant gliomas in adults. N Engl J Med 359: 492-507, 2008.

2. Khasraw M and Lassman AB: Advances in the treatment of malignant gliomas. Curr Oncol Rep 12: 26-33, 2010. 
3. Yu Y, Ramena G and Elble RC: The role of cancer stem cells in relapse of solid tumors. Front Biosci (Elite Ed) 4: 1528-1541, 2012.

4. Binda E, Visioli A, Reynolds B and Vescovi AL: Heterogeneity of cancer-initiating cells within glioblastoma. Front Biosci (Schol Ed) 4: 1235-1248, 2012.

5. Reya T, Morrison SJ, Clarke MF and Weissman IL: Stem cells, cancer, and cancer stem cells. Nature 414: 105-111, 2001.

6. Malik B and Nie D: Cancer stem cells and resistance to chemo and radio therapy. Front Biosci (Elite Ed) 4: 2142-2149, 2012

7. Beier D, Schulz JB and Beier CP: Chemoresistance of glioblastoma cancer stem cells - much more complex than expected. Mol Cancer 10: 128, 2011.

8. Hermisson M, Klumpp A, Wick W, et al: $\mathrm{O}^{6}$-methylguanine DNA methyltransferase and p53 status predict temozolomide sensitivity in human malignant glioma cells. J Neurochem 96: 766-776, 2006

9. Hegi ME, Liu L, Herman JG, et al: Correlation of $\mathrm{O}^{6}$-methylguanine methyltransferase (MGMT) promoter methylation with clinical outcomes in glioblastoma and clinical strategies to modulate MGMT activity. J Clin Oncol 26: 4189-4199, 2008.

10. Blough MD, Westgate MR, Beauchamp D, et al: Sensitivity to temozolomide in brain tumor initiating cells. Neurooncology 12: 756-760, 2010

11. Spiegl-Kreinecker S, Pirker C, Filipits $\mathrm{M}$, et al: $\mathrm{O}^{6}$-methylguanine DNA methyltransferase protein expression in tumor cells predicts outcome of temozolomide therapy in glioblastoma patients Neurooncology 12: 28-36, 2010.

12. Kato T, Natsume A, Toda $\mathrm{H}$, et al: Efficient delivery of liposome-mediated MGMT-siRNA reinforces the cytotoxity of temozolomide in GBM-initiating cells. Gene Ther 17: 1363-1371, 2010.

13. Ikushima $\mathrm{H}$, Todo $\mathrm{T}$, Ino $\mathrm{Y}$, Takahashi $\mathrm{M}$, Miyazawa $\mathrm{K}$ and Miyazono K: Autocrine TGF-beta signaling maintains tumorigenicity of glioma-initiating cells through Sry-related HMG-box factors. Cell Stem Cell 5: 504-514, 2009.

14. Matsuda K, Sato A, Okada M, et al: Targeting JNK for therapeutic depletion of stem-like glioblastoma cells. Sci Rep 2: 516, 2012.

15. Sato A, Sunayama J, Okada M, et al: Glioma-initiating cell elimination by metformin activation of FOXO3 via AMPK. Stem Cells Transl Med 1: 811-824, 2012.

16. Hart MG, Garside R, Rogers G, Stein K and Grant R: Temozolomide for high grade glioma. Cochrane Database Syst Rev 4: CD007415, 2013.

17. Hegi ME, Diserens AC, Godard S, et al: Clinical trial substantiates the predictive value of O-6-methylguanine-DNA methyltransferase promoter methylation in glioblastoma patients treated with temozolomide. Clin Cancer Res 10: 1871-1874, 2004.

18. Sato A, Sunayama J, Matsuda K, et al: MEK-ERK signaling dictates DNA-repair gene MGMT expression and temozolomide resistance of stem-like glioblastoma cells via the MDM2-p53 axis. Stem Cells 29: 1942-1951, 2011.

19. Ciceroni C, Bonelli M, Mastrantoni E, et al: Type-3 metabotropic glutamate receptors regulate chemoresistance in glioma stem cells, and their levels are inversely related to survival in patients with malignant gliomas. Cell Death Differ 20: 396-407, 2013.

20. Persano L, Pistollato F, Rampazzo E, et al: BMP2 sensitizes glioblastoma stem-like cells to Temozolomide by affecting HIF-1alpha stability and MGMT expression. Cell Death Dis 3: e412, 2012.

21. Siebzehnrubl FA, Silver DJ, Tugertimur B, et al: The ZEB1 pathway links glioblastoma initiation, invasion and chemoresistance. EMBO Mol Med 5: 1196-1212, 2013.
22. Ohba S, Hirose Y, Kawase T and Sano H: Inhibition of c-Jun N-terminal kinase enhances temozolomide-induced cytotoxicity in human glioma cells. J Neurooncol 95: 307-316, 2009.

23. Kanzawa T, Germano IM, Kondo Y, Ito H, Kyo S and Kondo S: Inhibition of telomerase activity in malignant glioma cells correlates with their sensitivity to temozolomide. Br J Cancer 89: 922-929, 2003

24. Aghi M, Rabkin S and Martuza RL: Effect of chemotherapyinduced DNA repair on oncolytic herpes simplex viral replication. J Natl Cancer Inst 98: 38-50, 2006.

25. Nadkarni A, Shrivastav M, Mladek AC, et al: ATM inhibitor KU-55933 increases the TMZ responsiveness of only inherently TMZ sensitive GBM cells. J Neurooncol 110: 349-357, 2012.

26. Miyazawa K: A negative regulator or just an unconcerned passerby: phosphoinositide 3-kinase signalling in IL-12 production. J Biochem 152: 497-499, 2012.

27. Jemaa M, Vitale I, Kepp O, et al: Selective killing of p53-deficient cancer cells by SP600125. EMBO Mol Med 4: 500-514, 2012.

28. Schmidt M, Budirahardja Y, Klompmaker R and Medema RH: Ablation of the spindle assembly checkpoint by a compound targeting Mps1. EMBO Rep 6: 866-872, 2005.

29. Kohsaka S, Wang L, Yachi K, et al: STAT3 inhibition overcomes temozolomide resistance in glioblastoma by downregulating MGMT expression. Mol Cancer Ther 11: 1289-1299, 2012.

30. Bocangel D, Sengupta S, Mitra S and Bhakat KK: p53-mediated down-regulation of the human DNA repair gene $\mathrm{O}^{6}$-methylguanine-DNA methyltransferase (MGMT) via interaction with Spl transcription factor. Anticancer Res 29: 3741-3750, 2009.

31. Boldogh I, Ramana CV, Chen Z, et al: Regulation of expression of the DNA repair gene $\mathrm{O}^{6}$-methylguanine-DNA methyltransferase via protein kinase C-mediated signaling. Cancer Res 58: 3950-3956, 1998.

32. Pyko IV, Nakada M, Sabit $\mathrm{H}$, et al: Glycogen synthase kinase 3beta inhibition sensitizes human glioblastoma cells to temozolomide by affecting $\mathrm{O}^{6}$-methylguanine DNA methyltransferase promoter methylation via c-Myc signaling. Carcinogenesis 34 : 2206-2217, 2013

33. Lavon I, Fuchs D, Zrihan D, et al: Novel mechanism whereby nuclear factor kappaB mediates DNA damage repair through regulation of $\mathrm{O}(6)$-methylguanine-DNA-methyltransferase. Cancer Res 67: 8952-8959, 2007.

34. Chuang JY, Wang YT, Yeh SH, Liu YW, Chang WC and Hung JJ: Phosphorylation by c-Jun NH2-terminal kinase 1 regulates the stability of transcription factor Spl during mitosis. Mol Biol Cell 19: 1139-1151, 2008.

35. Wang SA, Chuang JY, Yeh SH, et al: Heat shock protein 90 is important for Spl stability during mitosis. J Mol Biol 387: 1106-1119, 2009

36. Noguchi K, Kitanaka C, Yamana H, Kokubu A, Mochizuki T and Kuchino Y: Regulation of c-Myc through phosphorylation at Ser-62 and Ser-71 by c-Jun N-terminal kinase. J Biol Chem 274: 32580-32587, 1999.

37. Kitanaka C, Sato A and Okada M: JNK signaling in the control of the tumor-initiating capacity associated with cancer stem cells. Genes Cancer: Jan 22, 2013 (Epub ahead of print). doi: $10.1177 / 1947601912474892$.

38. Yoon CH, Kim MJ, Kim RK, et al: c-Jun N-terminal kinase has a pivotal role in the maintenance of self-renewal and tumorigenicity in glioma stem-like cells. Oncogene 31: 4655-4666, 2012. 